Note: This work is currently in publishing in 'L'immagine contemporanea: natura, forme, valori'

This is a pre-print version.

Cite as: Denicolai, L. (2020). The robotic gaze: A hypothesis of visual production using technological image/thinking. MediarXiv preprint.

\title{
The robotic gaze: A hypothesis of visual production using technological image/thinking
}

\section{Lorenzo Denicolai}

\section{Introduction}

The relationship between man and robots, as a technological form, has been depicted many times over the years in a variety of narrative media. The earlest known of these depictions is R.U.R. by Czech playwright Karel Capek (1920): Robots created in the laboratory of Dr. Rossum are a form of primitive hybridization between human genes and technological elements, creating a sort of cyborg ante litteram. Since then, robots have been portrayed in many literary works-Isaac Asimov ${ }^{1}$ dedicated his collection The Complete Robot to these machines, and Philip K. Dick's Do Androids Dream of Eletric Sheep is one of the most famous of these stories. In the same way, cinema has also often used man-robot relationships as an inspiration, highlighting and translating into images some of the natural inclinations of humanity to place (conditional) confidence in its potential substitute simulacra. Some of the most famous of these films include Westworld (Crichton, 1973), The Black Hole (1979, Nelson), Terminator (1984, Cameron), Bicentennial Man (1999, Columbus), Wall-E (2008, Stanton), Robot \& Frank (2012, Schreier), and Ex Machina (2015, Garland). To these, it is important to add the disturbing intelligence of HAL9000 in 2001: A Space Odyssey (Kubrick, 1969), the dystopic atmosphere of Blade Runner (1982, Scott), the Matrix trilogy (1999-2003, Wachowski siblings), as well as some television serials, such as Battlestar Galactica (2004-2009, Moore), Black Mirror (2011-, Brooker), and older series such as The Six Million Dollar Man (1974-1978), in which one could glimpse the precursor of the cyborg (Haraway, 2006; Clark, 2004; Hansen, 2004; 2006; Hayles, 2006; Verbeek, 2008). All the narratives ${ }^{2}$ cited here also present forms of a possible mirroring between the behavior of humans and robots, an element that reinforces the search for a relationship between these mechanical entities and human ontology.

This aspect is manifested in both the construction of narratives and characters (for example, in cases where the robot/humanoid is an ally or an opponent of humans) and at a metanarrative and intertextual level, i.e., with more or less explicit references to the real body of cinema. Susan Sobchack, for example, pointed out that Wall-E is a "cinephilic homage to photochemical cinema [...] through its remarkable, dialogue-free, 'silent' first act, its explicit modeling of its quasi-mechanical protagonist on Buster Keaton and Charlie Chaplin, and its often hilarious citations of science fiction movies both classic and contemporary" (2009, p. 379), highlighting a paradoxical performative quality that may hide another aspect of Wall-E's emotional evocation of Chaplin's and Keaton's many mannerisms. In general, all of the cinematographic works mentioned here are responses to the need to focus on robots' ability to see: all of the entities involved in these different productions are able to see, perceive, and cognitively process reality, and therefore to propose their own point of view. I propose a heuristic hypothesis that the use of some robots currently on the market - especially those designed for educational and social use - can also provide their own interpretations of reality through their ability to see, which may also give rise to a deeper relationship with humans. Before I begin, however, it is important to briefly describe precisely what I mean by the idea of a "human-robot relationship."

\section{A relationship between two living species}

Nowadays, the question of the relationship between humans and machines is expressed mainly in terms of human-robot interaction and is being explored in a wide range of academic literature, ranging from the field

\footnotetext{
${ }^{1}$ One of the first films inspired by Asimov's works was The Forbidden Planet (Wilkox, 1956), followed by The Invisible Boy (Hoffman, 1957), both of which starred Robby the Robot.

${ }^{2}$ Cf. Payr (2019).
} 
of psychology to computer science and artificial intelligence (AI). Its portrayals range from the purely sociological to pedagogical experimentation and assistance for people with disabilities or motor and cognitive deficits due to age. I shall limit myself here to mentioning two of the numerous international studies and conferences on the subject-the International Symposium in Robot and Human Interactive Communication hosted by the IEEE and the International Conference on Human-Robot Interaction hosted by the ACM/IEEE - as multidisciplinary assessments of the vast impact that human-robot interaction is having on everyday life. For the purposes of this contribution, I prefer to address the issue along two lines that are slightly different from those traditionally proposed by the scientific institutions mentioned above. First of all, I think that the human-robot relationship can to some extent respond to the model offered by Floridi (2014) in his reading of a "in-betweenness technology" (2014, chapter 2) - that is, a robot can serve as an intermediary between a human-user and the environmental context. Robot and human can act reciprocally, modelling and motivating one another's reactions using technology: "what the sun does is to prompt the development and then the wearing of the hat" (Ibid.). In order to define this technology and its relationship with humanity, Floridi identified a prompter to motivate this relationship, which can be modelled as follows:

user $\leftarrow$ technology $\rightarrow$ prompter

This scheme has three orders of application. The first considers technology as positioned between humanity and nature (nature, being natural, suggests the use of an axe as a tool to act on wood); the second replaces nature with another technology (e.g., the engine acts on the car); the third also replaces the user, but in this case an exclusive relationship exists between technologies - for example, a robot (a user-driven technology) using a pair of scissors (technology) to act on cloth (the technology prompter). ${ }^{3}$ This perspective, which in some ways recalls the approach of the so-called Theory of Material Engagement (Malafouris, 2013; 2018), can, in certain situations, cast the robot in the roles of both technology and prompter, just as the user can in turn take on the robot's identity in cases of a particular embodiment of its intentionality as a modality to reflect him- or herself in the robotic body (Verbeek, 2008). Therefore, if the user-human relates to the robot as a technology, he or she can use it in various ways; for example, it can be used to extend perception, such as increased vision, or as an embodiment of robotic touch. Such uses allow humans to intervene in some way in the interface of the robot, although this relies on the formulas of esoteric algorithmic actions. Hypothetically, if the relationship is of the third order, the user would instead become a technology (Parisi, 2019) and, as a medianthrope (Denicolai, 2018), through a reciprocal and deep incarnation, would also connect with the robot-technology to respond to a third technology-prompter. Thus, the relationship would give the user an extension into the body of the robot, which would in turn be embodied in the body-scheme of the user. The question of robotic embodiment in the user can also be read along the lines dictated by, among others, Besmer (2015): elaborating on the work of Merleau-Ponty, Besmer underlined how "[the] sense of being 'in' or 'inhabiting' the remote body of the robot" can be defined as a "robotic re-embodiment" (2015, 57). This "living" condition translates itself into the user's need to be immersed in the body of the robot and the simultaneous necessity for the robot to become transparent, creating a sort of medial immediacy that allows the user to perceive the remote perception as his own. This reflective mode presents a further dichotomy, which Parisi (2019) synthesized as an oppositional pair extension/reduction, according to which human cognition, even if extended by a technological medium, would remain limited by the dimensionality of the medium itself. Besmer pointed out that in reality, with a few exceptions, robotic reembodiment in the body of the robot is not yet able to return proprioceptive feedback. It is currently confined to postponed visual perceptions ${ }^{4}$ of the environment in which it operates remotely, to the point that "the level

\footnotetext{
${ }^{3}$ Examples are freely taken from the author (2014).

${ }^{4}$ Besmer refers to a robotics "remote", meaning that it is equipped with its own body and can be remotely controlled by its operator. Recent studies in the field of medical engineering have developed artificial limbs capable of tactile
} 
of bodily integration seems limited to that of the body image" (68) - that is, "a complex set of intentional states and dispositions - perceptions, beliefs, and attitudes - in which the intentional object is one's own body" (Gallagher, 2005, p. 25). The fact that the relationship of embodiment is confined to an exclusively visual context is relevant to the second hypothesis of the relationship between human and robot, which is based on the concept of the Foucauldian dispositive, itself based on the re-reading offered by Albera and Tortajada (2015) that was also elaborated on by D'Aloia and Eugeni (2017). ${ }^{5}$ Albera and Tortajada introduced the dispositive-episteme, a relational scheme that implies a continuous exchange between the heterogeneous elements that comprise it. Although they focus on the spectator-apparatus-representation relationship, the relationship itself can also be read as a further form of reciprocal incarnation between man and robot (Gordon, 2019) - a relationship that can produce media and visual objects and generate the necessary conditions for a user to become a spectator. This is because "the constitution of a body of knowledge associated with viewing and listening dispositives may be defined as unthinkable without linking these dispositives and a form of experience or practice" (Albera, Tortajada, 2015, 41-42). The human-robot relationship can thus also be understood as a productive relationship arising from a performative experience in progress, which is a direct result of the mutual fusion between these two elements. It translates itself into the ability of the robot's vision and potential to build meaning using the images it perceives and produces. Here, I am limiting myself to providing some theoretical lines of reference; below I offer some examples of relational forms and the possible resulting visual productions.

\section{Visions, relations, and technological images}

The robotic vision question is part of a wider scientific debate concerning vision mediated by technology, ${ }^{6}$ vision that can occur through the relationship between a human and a particular object, as well as with an artifact that can modify the human condition of departure. According to Verbeek $(2005 ; 2008)$, the relationships of intentionality that are established in the human-technology union-that is, the two entities often considered to be merged in the figure of the cyborg - also need to be investigated. I will return to this subject shortly in considering the relationship between the human-user and the humanoid social robot "Pepper" as potentially belonging to Floridi's (2014) third relational order. Meanwhile, the technological vision is to be understood as a sort of strengthening of human perceptive abilities: It is a type of machinic vision, ${ }^{7}$ a gaze that the machine manages for and with the human, as well as a way of translating a mechanical perception that is thereby rendered significant for human reception and cognition. However, "the machinic vision simply expands the range of perception well beyond the organic-physiological constraints of human embodiment" (Hansen, 2004, p. 99). The significant translation of an image as the result of a humanrobot connection is also underlined by Johnston's (1999) reading of machinic vision, as it "presupposes not only an environment of interacting machines and human-machine systems but a field of decoded perceptions that, whether or not produced by or issuing from these machines, assumes their full intelligibility only in relation to them" (p. 27). Building on Deleuze's theory, Johnston argued that the brain would tend to become a "deterritorialized organ", to the advantage of an "extended condition of visuality" (45), favoring once again the hypothesis of a collaboration with an apparatus which, in reality, would result from a reciprocal incarnation of human-robot. ${ }^{8}$ In this way it is possible to presume that the technological eye of the robot

perception that can stand in for the user's perception of touch. For further information, please refer to the European project DeTOP (available at: http://www.detop-project.eu/, last accessed on October 20, 2019).

${ }^{5}$ On the dispositive (and apparatus), in addition to the fundamental references for the disquisitions of Benjamin, Balázs, Moholy-Nagy, Foucault, and Deleuze, here I am referring to Agamben (2006), Bussolini (2010), Casetti (2015), Pinotti e Somaini (2016), and Somaini (2016).

${ }^{6}$ There is a vast amount of literature available on this question; see, for example, Hansen (2004; 2006), Manovich (2001; 2013), and Mitchell (2005; 2017), for some of the most pertinent discussions of the crisis between analytic and digital technology used to produce images in the post-photographic era.

${ }^{7}$ Cf. Virilio (1994).

${ }^{8}$ In his reading of the new media, Hansen believed that "in this posthuman perceptual regime, the selection of information is no longer performed exclusively or even primarily by the human component (the body-brain as a center 
(Arcagni, 2018) works autonomously and "reads" reality in the form of data and digital detections using "sightless vision" (Virilio, 1994, p. 59) - vision that must then be decoded to make the perceived elements accessible to human cognition. The status of the vision and of the technological images derived from humanrobot dialects would likewise require translation, also in the sense understood by Lévi-Strauss in his observations on the concept of "meaning": that is, the mechanical perception must be made comprehensible and evident for human cognition (Heidegger would say "to show it off").

I consider the now famous image of the black hole captured by the Event Horizon Telescope ${ }^{10}$ in April 2019 as belonging to the category of technological images (Fig. 1). It is the result of machinic vision, obtained using eight radio telescopes working in concert that provided a total of 5 petabytes of data, which was then reworked and translated by a supercomputer into the image that was broadcast by the media. ${ }^{11}$

\section{[Fig. 1]}

We may now be faced with what Baudrillard defined as a simulacrum of coding: Something that is result of the illusion of total detachment from reality and yet built exclusively using binary computer code. In this case there is no direct reference, so to speak, to the product obtained; however, the image of the black hole falls fully within a form of post-modern/post-medial (and, in part, post-human) vision that Verbeek (2008), elaborating on Ihde (1990), described as the result of a technological mediation. ${ }^{12}$ In a sort of radical mediation $a ̀$ la Grusin, the requirement for a realistic perception is rendered irrelevant, as "the 'original' which has to be represented cannot be known directly at all, but only through mediation" (2008, p. 4). As well as the real, it offers something that is the result of the human-machine relationship. It is a surplus image, as Mitchell (2017) put it, which to some extent exists beyond the physical, as if it were an image set in a picture: the image of the black hole would exist even at a different level, despite not being visible without a technological intervention to restructure it into a picture. The latter would be able to respond to semiological levels of the human context. Finally, this image/picture would respond to the need for sharing between human and non-human, united by the bit, the unit of measure that Longo and Vaccaro (2013), among others, asserted as a link in the post-human and cyborg era. Similarly, in the field of industrial robotics - particularly educational robotics - many types of mini-robots have the ability to see coding (for example, in the form of colored lines) using their sensors, translate it into action, and thus react with their own movements, so to

of indetermination)" (2004, p. 99). This argument seems to point to a potential collaboration between human and nonhuman technology.

9 "In semantics there is a curious fact: the word 'meaning' is probably [...] the one whose meaning is most difficult to establish. The word 'meaning' indicates the possibility that data of any kind can be translated into another language" (Lévi-Strauss, 2010, p. 25-26; English translation mine). This "translation" would also be available as a form of Verbeek's "hermeneutic intentionality": "hermeneutic relations always involve a technologically generated representation of the world, which inevitably is the product of a specific technological directdness at the world. The composition of human intentionality and technological intentionality is directed at making accessible ways in which technologies 'experience' the world" (2008, 393).

10 The image is available at https://eventhorizontelescope.org/blog/first-ever-image-black-hole-published-eventhorizon-telescope-collaboration (accessed October 22, 2019).

${ }^{11}$ According to Arcagni, "Computer Vision can be understood as the computer process that converts a numerical representation of the image into a symbolic representation oriented towards form" because it "acquires, processes, analyses, understands and interprets digital images" (2018, chapter 2; English translation mine). Similarly, at a conference in Milan in May 2019, Somaini pointed out that today the so-called machine-readable vision is able to read images that are "radically invisible", i.e., images that in reality are never visible to the human eye but only to the technological eye of the machine.

${ }^{12}$ Verbeek, citing Ihde, described such mediations as follows: "Contemporary technologies like radio telescopes make visible realities to us that cannot be perceived without these mediations. [...] the realities revealed by these mediating technologies do not have an equivalent to the naked eye" $(2008,3)$. 
speak, to input seen with technological eyes: in this way, the robot is able to perform (a real type of action) and to initiate, theoretically, forms of collaboration and significant co-construction. ${ }^{13}$

\section{Collaborative relationships and autonomous visions}

The relationship between humans and robots can therefore constitute an apparatus of vision. This collaboration, moreover, allows for a deepening of experiential, aesthetic, and obviously visual production, as well as consideration of the quality of the images that the human-robot dispositive can display - not to mention those they can generate. A better illustration of what I mean is some of the interactions that are possible with the humanoid Pepper. ${ }^{14}$ Among other functions, Pepper is able to perceive and recognize people using facial recognition, and can interact with those around it and with its environment. It can also see with its own direct perception, a sort of co-agent relationship with the environment that surrounds it (Gibson, 1986; Boccignone, 2001). What it perceives can be seen in real time by the human eye on the screen of a PC to which the humanoid is connected via wi-fi. I will focus in particular on two possible scenarios. The first of these scenarios is that of images realized with what is called depth vision (Fig. 2): Pepper can see, read, and make available to our perception the depth of the environment and the various distances between the objects present in a given space.

[Fig. 2]

Pepper is able to make explicit the spatial relationship between the different objects and elements present in an environment. Unlike direct perception-human vision, in other words-Pepper can make perceivable to our eyes not only how objects appear (i.e., how they are shown), but also where they are shown and their distance from the human viewing its visual representations (a real robotic point of view). In addition to the technological image mentioned above, I believe that we can also hypothesize the essence of an aniconic image. After all, the reproduction of space with depth vision seems to correspond to the definition provided by Pinotti, who argued that "an-icons are images becoming environments provided with agencies and affordances: in corresponding to these stimuli, the spectator becomes a veritable 'experiencer"' $(2017,3)$. The power of the robot to transfer to our sight a different reading of media or the environment and to make it somehow livable seems obvious. This ability means that not only is the body a place of images (Belting, 2001), but that the image can also be considered a place for the body, in a co-agent penetration between entities. Moreover, by an extreme paradox, we now have the ability to see through the eyes of humanoids, almost as if we could be immediately incarnated in the body of a robot. This is not only a matter of technological embodiment in our body, but also of the inverse process - that is, of our mirroring in the machine, in a sort of reciprocal and continuous adjustment. Massumi's (1998) ${ }^{15}$ words on depth perception are particularly relevant to the action that can be taken through Pepper's gaze: "Depth perception is a habit of movement. When we see one object at a distance behind another, what we are seeing is in a very real sense our own body's potential to move between the objects or to touch them in succession" (p. 21). The implication of this statement is that Pepper can also be an avatar, an extension capable of allowing us to deeply perceive an experience of a spatial vision. While we saw something with "our" body, ${ }^{16}$ including its

\footnotetext{
${ }^{13}$ For example, the robot Ozobot and most Lego Mindstorms are equipped with a follower reader, as well as the normal forms of automatism present in companies and industries. In some experiments carried out by the research group belonging to the Luciano Gallino Laboratory of Behavior Simulation and Educational Robotics, we have created audiovisual products in which robots are the protagonists of narrative actions. I refer to Denicolai et al. (2017). For other forms of collaborative co-constructions, see Sandry $(2015 ; 2017)$ and Cizek, Uricchio and Wolozin (2019).

${ }^{14}$ The Pepper robot is a humanoid created by SoftBank, and was conceived and designed as a social robot. In Japan it is already being used to help care for the elderly. For further information, see https://www.softbankrobotics.com/ emea/en/pepper (accessed October 24, 2019).

${ }^{15}$ Hansen (2004) used this quotation in his accurate disquisition on the automation of sight (pp. 92-123; here, p. 108).

${ }^{16}$ I am indirectly referring to Hansen (2001).
} 
physicality and materiality, ${ }^{17}$ a body external to ours, in a condition of heteroflexivity, would change us into technological post-humans. We would become a third relational order of Floridi, where the technological element would use its declination measurements to act on other technologies.

The second case is based on the assumption that Pepper is a medium of vision but one that, being endowed with artificial intelligence and machine learning, also has the potential to have an autonomous life. In practice, Pepper, properly set, is free to look at what catches its attention and to record every object it perceives. This aspect reveals a further relational level: If the technological image and the translated image perceptible by human cognition via the intervention of computing is oriented to the visual formalization of binary inputs (e.g., in a second relational order of Floridi), the intelligence of the machine allows a sort of true bidirectional relationship that is, as outworn as the term may seem, equal. This proposition approaches the hypothesis of a condition of cognisphere (Hayles, 2006), where the human enters into a mutual exchange with the robot, initiating processes of co-evolution and co-production between the species (Hoelzl and Marie, 2016). Moreover, if we consider Schröter and Ernst's (2018) reading that AI is to be considered a form of medium, once again it would be possible to maintain the relational connection: If everything is mediated (we are in a radical mediation), Pepper's AI (i.e., its way of thinking, processing data with machine learning, and behaving) also acts as a medium, something that stands between us and the world (Parisi, 2019). Verbeek's technological mediation in this case would originate a form of "constructive intentionality"- that is, "a technology-world relation [...] [that] implies a double intentionality [...]; one of technology toward 'its' world, and one of human beings toward the result of this technological intentionality. Humans are directed at the ways in which a technology is directed at the world" (2005, p. 6). The case illustrated in Fig. 4 is a still image of what Pepper sees with its computational eyes during an interaction (speaking with a person). Pepper gives us its own point of view, which on closer inspection could be reread in the sense of the bodysensor proposed by Eugeni $(2013)^{18}$ in his definition of the first-person shot. In this sense Pepper can be both the subject of the action and the camera that relays the (its own) performance, in an overlapping of functions between the filmic and the pro-filmic that refers, among others, to avant-garde instances, of which Vertov's Kino-Eye (1924) must be mentioned, or the machinic power detected by Epstein (1946), among others. Pepper would thus be both the subject and the object of itself, a digital form of a machinic subjectivity, ${ }^{19}$ almost an "animistic medium" (Castro, 2016, p. 248) ${ }^{20}$ able to play the role that Latour synthesized in the concepts of "quasi-object" and "quasi-subject": ${ }^{21}$ A "hybrid being, caught within a constant exchange of properties with other human and non-human 'actans"” (Castro, 2016, p. 250). According to Castro's reading, it emerges not only that the camera (in our case, the robot and its eyes) is embodied in an animistic medium (which Castro identified as the cinema), but also that the camera is the bearer of an intentionality, a form of presence that is obviously mediated (Verbeek, 2008) and autonomously provides us with a vision of the context that has its own relationship with the environment. This relationship is represented to us in real time and transmits to us a sort of robotic affective agent with space. Pepper thus seems to assume a life of its own,

\footnotetext{
${ }^{17}$ For further discussion of the relationship with the avatar, see Couchot (2019) and Riva (2009) on the issue of mediated presence.

${ }^{18}$ Discussing the first person shot, Eugeni (2013) specifies that, unlike the traditional subjective, in this case there is no clear distinction between the framed subject and the object, i.e., the camera offers the gaze, but the two "form a unitary entity that assumes the characters of one and the other and that can oscillate from one to the other of the two poles that constitute it" (p. 21. English translation mine). See also Eugeni (2016).

19 About the machinic soul: "The cinematograph is among the still partially intellectual robots that [...] shapes representations - that is, thought - in which we discern the primordial framework of reason: the three categories of extension, duration, and causation. [...] [T] he cinematograph inscribes its own character within its representations of the universe with such originality that it makes this representation not simply a record or copy of the conceptions of its organic mastermind, but indeed a differently individualized system, partly independent" (Epstein, 1946/2014). See also Turvey (1998).

${ }^{20}$ I wonder if the animistic medium, in its "materiality" of cinema, as Castro (2016) put it, cannot be reinterpreted yet as a dispositive apparatus and therefore as another heterogeneous relationship that allows vision.

${ }^{21}$ These concepts are presented in Latour (1993) and taken up in Castro's in-depth study (2016).
} 
perhaps a kind of consciousness, ${ }^{22}$ as it explores the environment. It is able to live in this space, to replay and remediate it on the PC screen it is connected to via wi-fi and, at the same time, to record the object of its vision, as a more traditional documental technology, thus allowing us to objectify our perception. In a kind of technological oculacentrism and equal relationship, the robot seems to support and enhance the vision, becoming from time to time physical avatar, simulacral substitute, and ultimately ally in a visual and relational reading of reality.

\section{Bibliography}

- Agamben, Giorgio. Che cos'è il dispositivo?. Roma: Nottetempo 2006.

- Albera, Francois and Tortajada Maria. "The dispositive does not exist!". In Cine-Dispositives: Essays on Epistemology of Films, edited by Francois Albera and Maria Tortajada, 21-44. Amsterdam: AUP 2015.

- Arcagni, Simone. L'occhio della macchina. Torino: Einaudi 2018.

- Belting, Hans. Antropologia delle immagini. Roma: Carocci 2001.

- Besmer, Kirk M. "What Robotic Re-embodiment Reveals about Virtual Re-embodiment: A Note on the Extension Thesis". In Postphenomenological Investigations, edited by Robert Rosenberger and Peter-Paul Verbeek, 55-73. London: Lexington Books 2015.

- Boccignone, Giuseppe. "Visione Artificiale". In Intelligenza artificiale. Manuale per le scienze umane, edited by Ernesto Burattini and Roberto Cordeschi, 137-170. Roma: Carocci 2001.

- Bussolini, Jeffrey. “What is a Dispositive?". In Foucault Studies (November 2010), no. 10: 85-107. https://doi.org/10.22439/fs.v0i10.3120.

- Casetti, Francesco. Galassia Lumière. Sette parole chiave per il cinema che viene. Milano: Bompiani 2015.

- Castro, Teresa. "An Animistic History of the Camera: Filmic Forms and Machinic Subjectivity". (2016): 247-255.

- Chella, Antonio and Manzotti Riccardo (eds.). "Machine Counsciousness: A Manifesto for Robotics". In International Journal of Machine Counsciousness 1 (2009), no. 1: 33-51. https://doi.org/10.1142/S1793843009000062

- Cizek, Katerina, Uricchio, William and Wolozin Sarah. "Part 6: Media Co-Creation with NonHuman Systems". In Collective Wisdom, edited by katerina Cizek and William Uricchio. (2019). https://wip.mitpress.mit.edu/pub/collective-wisdom-part-6?readingCollection=f7c1b7e5

- Clark, Andy. Natural-Born Cyborgs: Minds, Technologies, and the Future of Human Intelligence. Oxford: OUP, 2004.

- Couchot, Edmond. "Automatism, Autonomy and Aesthetics in the Performing Arts". In Leonardo 52 (June 2019), no. 3: 240-246. https://doi.org/10.1162/leon_a_01752

- D’Aloia, Adriano and Eugeni, Ruggero. Teorie del cinema. Il dibattito contemporaneo. Milano: Cortina 2017.

- Denicolai, Lorenzo, Grimaldi, Renato and Palmieri Silvia. "Videos, Educational Robotics and Puppets: an Experimental Integration of Languages". In END Proceedings, edited by Mafalda Carmo, 590-594. Lisbon: InSciences 2017.

\footnotetext{
${ }^{22}$ On this subject, I refer to Chella and Manzotti (2009), and Robitzski (2018).
} 
- Denicolai, Lorenzo. Mediantropi. Introduzione alla quotidianità dell'uomo tecnologico. Milano: FrancoAngeli 2018.

- Epstein, J. The Intelligence of a Machine, trans. Christophe Wall-romana, Minneapolis: UMP 2014.

- Eugeni, Ruggero. "Il First Person Shot come forma simbolica. I dispositivi della soggettività nel panorama postcinematografico". In Reti Saperi Linguaggi, 4 (2013), no. 2: 19-23. http://www.coriscoedizioni.it/wp-content/uploads/2013/12/Eugeni.pdf

- — "Remediating the presence. First person Shot and post cinema subjectivity". In Rimediazioni. Immagini interattive. Tomo 1, edited by Tiziana Migliore, 205-218. Roma: Aracne 2016.

- Floridi, Luciano. The Fourth Revolution: How the Infosphere is Reshaping Human Reality. Oxford: OUP 2014.

- Gallagher, Shaun. How the Body Shapes the Mind. New York: OUP 2005.

- Gibson, James J. The Ecological Approach to Visual Perception. London: Routledge 1986.

- Hansen, Mark B.N. New Philosophy for New Media, Cambridge: The MIT Press, 2004.

- - Bodies in Code: Interfaces with Digital Media, Abingdon: Routledge, 2006.

- Haraway, Donna. "The Cyborg Manifesto: Science, Technology, and Socialist-Femnism in the Late $20^{\text {th }}$ Century". In The International Handbook of Virtual Learning Environments, edited by Joel Weiss, Jason Nolan, Jeremy Hunsinger and Peter Trifonas, 117-158. Dordrecht: Springer, 2006

- Hayles, N. Katherine. "Unfinished Work: From Cyborg to Cognisphere". In Theory, Culture \& Society 23, (December 2006), no. 7-8: 159-166.

- Hoeltz, Ingrid and Rémi Marie. "Posthuman Vision". In Proceedings of the 22nd International Symposium on Electronic Art ISEA2016, 294-296.

- Ihde, Don. Technology and the Lifeworld: From Garden to Earth. Bloomington \& Indianapolis: IUP 1990.

- Johnston, John. "Machinic Vision". In Critical Inquiry 26 (Autumn 1999), no. 1: 27-48. www.jstor.org/stable/1344144.

- Latour, B. We Have Never Been Modern. Harvard: HUP 1993.

- Lévi-Strauss, Claude. Mito e significato. Cinque conversazioni radiofoniche. Milano: Il Saggiatore 2010.

- Longo, Giuseppe and Vaccaro, Andrea. Bit Bang. La nascita della filosofia digitale. Milano: Apogeo 2013.

- Manovich, Lev. The Language of New Media. Cambridge: The MIT Press 2001.

- - Software Takes Command. London: Bloomsbury 2013.

- Massumi, Brian. "Sensing the Virtual, Building the Insensible". In Architectural Design, 133, 68, 5/6, pp. 16-24, 1998.

- Mitchell, William J.T. What Do Pictures Want?: The Lives and Loves of Images. Chicago: UOC 2005.

- — Pictorial Turn. Saggi di cultura visuale, edited by Michele Cometa and Valentina Cammarata. Milano: Cortina 2017.

- Parisi, Francesco. La tecnologia che siamo. Torino: Codice 2019.

- Payr, Sabine. "In Search of a Narrative for Human-Robot Relationships". In Cyernetics and Systems: An International Journal 50 (2019), no. 3 281-299. https://doi.org/10.1080/01969722.2018.1550913.

- Pinotti, Andrea and Somaini Antonio, eds. Cultura visuale. Torino: Einaudi 2016.

- Pinotti, Andrea. "Self-Negating Images: Toards An-Iconology". In Proceedngs, n.d. Basel: MDPI 2017.

- Riva, Giuseppe. "Is presence a technology issue? Some insights from cognitive sciences". In Virtual Reality 13 (September 2009), no. 3: 159-169. https://doi.org/10.1007/s10055-009-0121-6 
- Robitzski, Dan. "Artificial Counsciousness: How to Give A Robot A Soul". In Futurism. https://futurism.com/artificial-consciousness

- Sandry, Eleanor. Robots and Communication. New York: Palgrave McMillian 2015.

- — "Creative Collaboration with Machines". In Philosophy and Technology 30 (September 2017), no. 3: 305-319. https://doi.org/10.1007/s13347-016-0240-4.

- Schroter, Jens and Ernst Christoph. "AI and the form of capital - Preliminary notes on the media culture of artificial intelligence". (2018). https://bonndoc.ulb.unibonn.de/xmlui/bitstream/handle/20.500.11811/1130/AI and the form of capital.pdf?sequence $=1 \&$ is Allowed $=\mathrm{y}$

- Sobchack Susan. "Animation and automation, or, the incredible effortfulness of being". In Screen 50 (Winter 2009), no. 4: 375-391. https://doi.org/10.1093/screen/hjp032.

- Somaini, Antonio. "Walter Benjamin's Media Theory: The Medium and the Apparat". In Grey Room 62 (February 2016), no. 62: 6-41. https://doi.org/10.1162/GREY_a_00188.

- Turvey, Malcom. “Jean Epstein's Cinema of Immanence: The Rehabilitation of the Corporal Eye". In October 83 (Winter 1998): 25-50. www.jstor.org/stable/779069.

- Verbeek, Peter-Paul. What Things Do: Philosophical Reflections on Technology, Agency, and Design. University Park: The Pennsylvania State University 2005.

- _ _ . "Cyborg Intentionality: Rethinking the phenomenology of human-technology relations". In Phenomenology and the Cognitive Sciences 7 (September 2008), no. 3: 387-395.

- Virilio, Paul. The vision machine. Bloomington \& Indianapolis: IUP 1994. 